\title{
SUPRACONDYLAR FEMORAL OSTEOTOMY FOR KNEE FLEXION CONTRACTURE RESULTING FROM POLIOMYELITIS
}

\author{
JOHN C. Y. LEONG, C. O. ALADE, DAVID FANG \\ From the Department of Orthopaedic Surgery, University of Hong Kong
}

\begin{abstract}
Supracondylar femoral osteotomy was performed on 82 patients between 8 and 25 years of age to correct knee flexion contractures of 15 to 95 degrees resulting from poliomyelitis. Seven who could not walk before the osteotomy could do so afterwards and 21 out of 22 patients were cured of a hand-knee gait. Thirty-four of 53 who walked with a caliper were able to discard it. Residual flexion contractures of 5 to 20 degrees were found in 12 patients, while genu recurvatum of 5 to 30 degrees occurred in 10 patients.
\end{abstract}

Joint contractures are common sequelae of many neuromuscular disorders. In patients with poliomyelitis flexion contracture of the knee is one of the commonest problems encountered. If the contracture is mild, and provided the opposite leg has relatively little motor paralysis, the patient walks with a hand-knee gait. If the contracture is more severe, the affected leg cannot bear weight, and if the opposite leg is also moderately paralysed, the ability to walk is lost.

It is our experience that in well selected cases, a single procedure to straighten the knee often leads to a dramatic improvement in function.

A review of the literature shows that many methods of correcting flexion contractures of the knee have been described. In 1900 Bradford and Lovett recommended hamstring tenotomy followed by manipulation. The results were poor in correcting deformity greater than $\mathbf{2 0}$ degrees, often with incomplete correction and a high recurrence rate within a short interval. In 1926 Yount demonstrated the importance of release of the tensor fascia lata. In 1929 Wilson recommended posterior capsulotomy as a preliminary to manipulation. These methods corrected mild deformity but in severe deformities often led to posterior subluxation of the tibia on the femur and a stiff and painful joint. In 1956 Clark recommended transfer of the hamstrings to the quadriceps but this is applicable only when the hamstrings are of nearly normal strength. Keats and Kambin (1962) have shown this procedure to be indicated in children with cerebral spastic paralysis.

In 1970 Conner described a technique using serial manipulations that prevented subluxation of the tibia, and also reported his experience with arthrodesis of the knee and supracondylar osteotomy in three cases. In 1977 Abraham, Verinder and Sharrard reported a 55 per cent success rate with the use of supracondylar femoral osteotomy to treat flexion contracture of the knee in patients with myelomeningocele.
Relatively little has been written of the role of operations on bone in the treatment of knee flexion contractures resulting from poliomyelitis. It is our experience that contractures of less than 15 degrees are easily corrected by passive stretching and serial casting. However, for any greater contracture conservative treatment is associated with incomplete correction and a high rate of recurrence. Complete correction, and even slight over-correction allowing the knee to lock in extension, is desirable in a poliomyelitic limb, especially when the power of the quadriceps is ineffective or absent. At the Duchess of Kent Children's Hospital, Hong Kong, we have used a one-stage supracondylar femoral osteotomy to obtain this correction. It is the purpose of this paper to review the results of this method of treatment.

\section{MATERIAL AND METHODS}

Between 1970 and 1978151 patients with knee flexion contractures resulting from poliomyelitis were treated at the Duchesss of Kent Children's Hospital, Hong Kong. Thirty-nine patients with contractures of less than 15 degrees were treated conservatively and have been excluded from this study. Thirty other patients were excluded either because the records were incomplete or the follow-up period was less than two years, or because multiple surgical procedures had been performed around the knee joint.

Eighty-two patients with a total of 89 knees were included in this study. There were $\mathbf{4 0}$ males and $\mathbf{4 2}$ females. Forty-one procedures were done on the right knee and 48 on the left. The age at operation ranged from 8 to 25 years with an average of 14 years and 9 months (Fig. 1). Thirty of the 82 patients could be classified as adults as they had radiographic evidence of closure of the lower femoral growth plate before operation.

The patients were selected for operation if the contracture hampered gait, prevented the fitting of a caliper or resulted in pressure sores. If they were unable to walk they had to have relatively normal power in the upper limbs, especially in the triceps. before surgery was contemplated.

The severity of flexion contracture of the knee ranged from 15 to 95 degrees with an average of 30 degrees. Fifty knees had a contracture greater than 25 degrees, and of these, 12 were greater than 40 degrees. Eighty-two supracondylar femoral osteotomies were

J. C. Y. Leong, FRCS, FRCSE, Head of Department C. O. Alade, MD, Former Research Associate

Department of Orthopaedic Surgery, University of Hong Kong, Hong Kong.

D. Fang, MCh(Orth), FRCSE, Lecturer

Requests for reprints should be sent to Professor J. C. Y. Leong.

() 1982 British Editorial Society of Bone and Joint Surgery 0301-620X/82/2042-0198 $\$ 2.00$ 


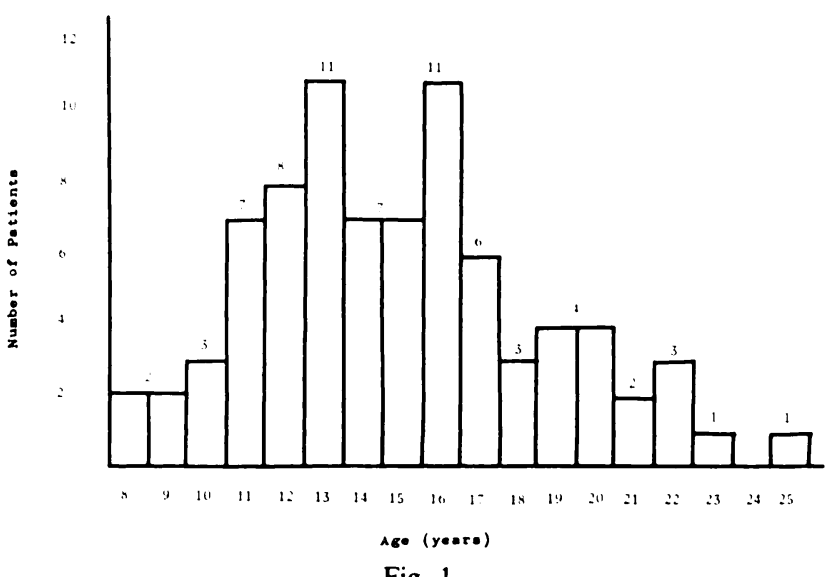

Fig. 1

Graph showing the age of patients at the time of operation.

performed as the only procedure for correction of the knee flexion contracture. In seven instances where flexion contracture exceeded 60 degrees, the osteotomies were preceded by a soft tissue release. The follow-up ranged from 2 to 10 years with an average of seven years and two months.

\section{SURGICAL TECHNIQUE}

The femoral osteotomy is done under a pneumatic tourniquet. A lateral approach is used. The tensor fascia lata is divided transversely if it is tight, as in Yount's procedure (Yount 1926). Care is taken not to enter the suprapatella pouch. The lower end of the femur is exposed subperiosteally. In the growing child, the growth plate is carefully identified and the osteotomy is made about 2.5 centimetres proximal to it. An anteriorly-based wedge is removed in the supracondylar region, leaving the posterior cortex intact. The wedge is then closed. No internal fixation is required when the osteotomy is done distally enough, as the bone is often osteoporotic and can easily be impacted. An intact posterior cortical hinge also adds to the stability. A suction drain is used routinely for the first $\mathbf{4 8}$ hours. External immobilisation with a long-leg plaster-of-Paris cast is adequate.

\section{POST-OPERATIVE REGIME}

The patients get up and walk on crutches. bearing no weight, between the fifth and seventh day. At two weeks the plaster cast is changed and the stitches removed. If the radiographs show incomplete correction. an additional manipulation can be carried out to obtain complete extension of the knee. Full weight-bearing is allowed at six weeks and immobilisation continued for 10 to 12 weeks, depending on the age of the patient. After removal of the splint the patients are kept in hospital under close supervision of a physiotherapist until the range of knee flexion is 90 degrees; thereafter they can be treated as out-patients. It usually takes two to three weeks to achieve $\mathbf{9 0}$ degrees of flexion.

\section{ILLUSTRATIVE CASE HISTORIES}

Case 1. A 16-year-old boy was first seen in 1971. He had a history of poliomyelitis, with residual involvement of both shoulders and both legs. For the past 12 years he had only been able to crawl. He had knee flexion contractures of 90 degrees in the left leg and 60 degrees in the right (Fig. 2). Posterior soft tissue release followed by serial plaster cast wedging reduced the contractures to 40 degrees and 35 degrees respectively. Supracondylar femoral osteotomy was then performed to correct the residual contractures to full extension. After the operation, he was fitted with bilateral long-leg calipers and was able to walk with two sticks (Fig. 3).

On final follow-up he had significant recurvatum when he stood without the calipers. He had no pain on walking. The recurvatum is unlikely to progress as he will have to wear the calipers for life.

Case 2. A 14-year-old girl was first seen in 1970. She gave a history of poliomyelitis at the age of two with residual involvement of the right

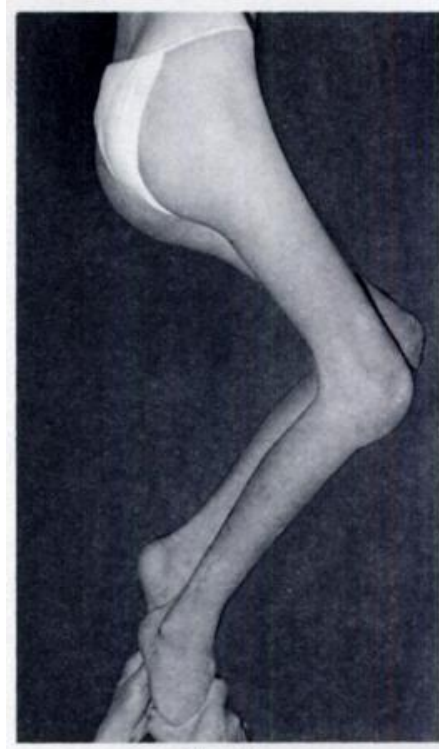

Fig. 2

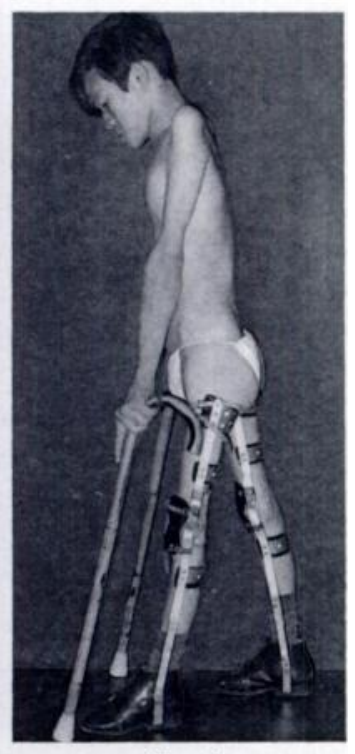

Fig. 3
Case 1. Figure2-View of both knees showing flexion contractures and the thickened skin over the front of the knees due to prolonged crawling. Figure 3-After correction of flexion contractures, the patient can walk with bilateral long-leg calipers and walking sticks.

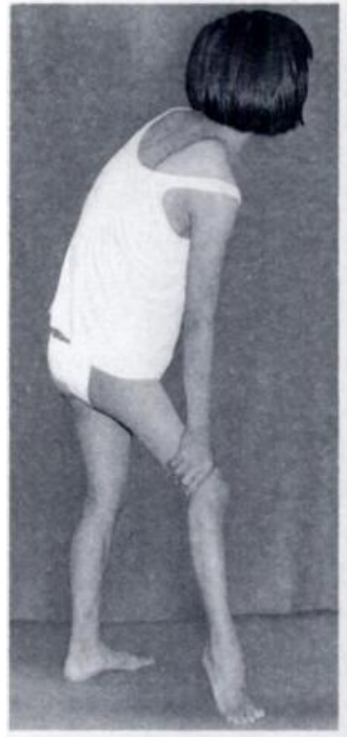

Fig. 4

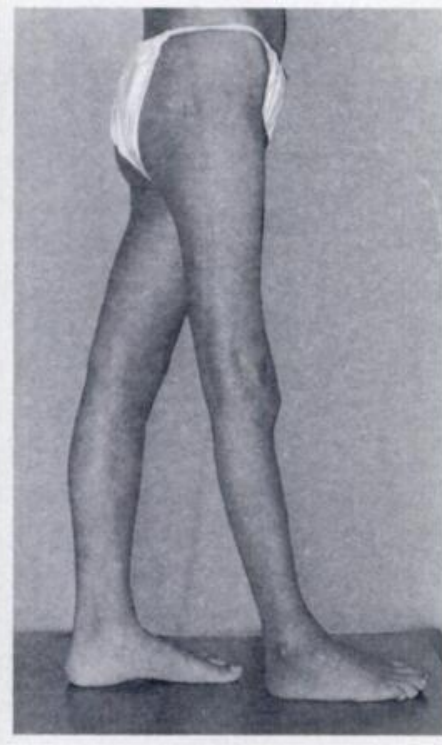

Fig. 5
Case 2. Figure 4-Photograph showing hand-knee gait before operation. Figure 5-Photograph showing full extension and absence of hand-knee gait after operation.

leg. She had a flexion contracture of 35 degrees and walked with a hand-knee gait (Fig. 4). After operation she walked without a hand-knee gait, had no pain. and could walk unlimited distances (Fig. 5).

\section{RESULTS}

Gait. Before the operation, seven patients could not walk. Fifty-three walked with long-leg calipers on one or both legs and more than half of these patients also required sticks or crutches. Twenty-two patients walked without calipers but had a hand-knee gait.

All seven who could not walk could do so after the 
operation, two being free of apparatus and five requiring the use of a caliper on one or both legs with or without crutches. Of the 53 who walked with a caliper, 34 were able to manage without a caliper on the operated leg. Of these 34 patients, 10 walked using sticks or crutches, and eight still wore a caliper on the other leg. All the 22 patients who were caliper-free before operation remained so afterwards, and 21 were rid of the hand-knee gait.

Correction, recurrence and recurvatum. During follow-up, 12 patients had a residual flexion contracture of between 5 and 20 degrees. On careful review, six of them had been incompletely corrected by the operation but in only five patients the contracture was considered sufficient to warrant a repeat procedure. This was done in four, as the fifth one refused further treatment.

One year after the initial operation, 10 patients demonstrated a recurvatum of between 5 and 30 degrees as shown on a lateral radiograph with the patient standing. There was no progression noted on further follow-up. Only one patient with 30 degrees of recurvatum was symptomatic and she remained in a caliper.

Range of movement. The range of flexion was between 90 and 120 degrees in 28 knees and between 120 and 155 degrees in 57 knees. Thirty nine out of 47 patients ( 83 per cent) operated on below 16 years of age could flex their knee to between 120 and 155 degrees, while eight out of 47 (17 per cent) had a range from 90 to 120 degrees. Of the 35 patients operated on at age 16 or older, 15 (42 per cent) had a range from 120 to 155 degrees, while 16 ( 45 per cent) had a range from 90 to 120 degrees. Four patients ended up with a stiff knee with less than 90 degrees of flexion.

Of 17 knees having a contracture of $\mathbf{4 0}$ degrees or more before operation, only four had a post-operative range of 120 to 155 degrees. The same range, however, was found in 53 out of 72 knees that had a contracture of less than 40 degrees. Of the four stiff knees, two had had a mild deformity before operation but the osteotomy had been complicated by infection. The other two knees had had a severe deformity of 90 degrees. In one the final range was only 35 degrees, and in the other 80 degrees.

Pain. None of the patients had pain before the operation. Post-operatively, there were six patients who had pain which affected walking. Two of these patients had less than 90 degrees of flexion, two had posterior tibial subluxation, and one had 30 degrees of recurvatum. The sixth patient was actually able to discard his long-leg caliper and we had grounds to suspect secondary gain as a reason for his claiming to have pain.

\section{Complications}

Infection. There were six cases of wound infection, three superficial and three deep. Deep infection resulted in markedly decreased range of joint movement. One had flexion to between 5 and 10 degrees, one to 45 degrees, and one from 10 to 45 degrees.

Nerve palsy. There were two cases of common peroneal nerve palsy which eventually resolved after about one year and two and a half years respectively.

Tibial subluxation. There were two patients whose radiographs showed posterior tibial subluxation; both had a significant amount of pain. They were aged 13 and 18 years at operation. The degree of initial flexion contracture was 60 degrees and 90 degrees respectively and both patients had undergone posterior soft tissue release including posterior capsulotomy. Supracondylar femoral osteotomy was performed to correct residual contractures of 30 and 45 degrees.

\section{DISCUSSION}

The walking ability of a patient with poliomyelitis depends mainly on the extent of the paralysis in the lower limbs. Patients with one normal or nearly normal leg, and some residual power in the antigravity muscles (gluteus maximus, quadriceps, and triceps surae) of the other lower limb, can walk without external support. If a flexion contracture develops in the knee of the affected limb, they often assume a hand-knee gait. If severe flexion contracture develops in the presence of a paralysed quadriceps, the affected knee buckles on weight-bearing, and walking is extremely difficult without external aid. Under the latter two circumstances, correction of the contracture, allowing full extension, or even slight hyperextension and locking of the knee, will lead to dramatic improvement. The hand-knee gait will be abolished, and any external aid can be discarded.

With bilaterally severely affected or flail legs, the patient will resort to crawling in the presence of knee flexion contractures. In these patients, correction of the contractures is an essential part of a programme aimed at walking with long-leg calipers.

Our results have shown that all seven who could not walk were able to do so after the operation, that 34 out of 53 who walked with calipers were able to discard the caliper on the operated leg, and 21 out of 22 patients were cured of the hand-knee gait. These results were achieved through the performance of a single procedure to correct the flexion contracture.

Some loss of movement is to be expected, as the osteotomy merely alters the arc of motion. However, the range after operation was satisfactory in most cases. Over half of the knees (57) had a range of flexion between 120 and 155 degrees. Patients older than 16 years of age, and contractures greater than $\mathbf{4 0}$ degrees, generally had a greater loss of movement. The main handicap appeared to be in patients whose homes were fitted with outdated toilets that necessitated squatting.

Weak or absent quadriceps with relatively strong hamstrings are a common finding in patients with knee flexion contractures due to poliomyelitis, although some 
contractures occur in flail limbs, probably as a result of posture. In the presence of an ineffective quadriceps, recurrence is common especially when the contractures are treated by conservative methods or by a soft tissue release. The reason may be that such methods often fail to obtain full extension.

Abraham et al. (1977), treating patients with a myelomeningocele, used as their main criterion of a successful operation a flexion contracture of less than 20 degrees at follow-up. We have little experience with myelomeningoceles, but certainly in patients with poliomyelitis the correction should aim at complete extension and even minimal hyperextension. The ability to lock the knee in full extension often permits freedom from the use of calipers. If calipers are required anyway because of extensive paralysis, a small amount of residual contracture may be permissible.

The recurrence rate was low in this series; in only 6 out of 89 was it greater than five degrees. There was, however, a recurvatum of more than five degrees in 10 patients. Although minimal hyperextension may even be desirable recurvatum beyond five degrees should be avoided. None of the knees with significant recurvatum progressed and only one patient had pain.
Conner (1970) emphasised that congruous correction is important to achieve a painless knee with a useful range of movement. None of our patients had pain before operation but five patients, two of whom had posterior tibial subluxation, had significant pain afterwards. In these two patients, the tibial subluxation was probably caused by serial wedged plasters following posterior capsulotomy. Supracondylar osteotomy naturally did not improve the congruity and in retrospect was not indicated. In such severe cases perhaps hamstring tenotomy with division of the iliotibial band as described by Conner (1970) should be used to achieve as much congruous correction as possible before osteotomy is contemplated.

In conclusion, we believe that, in limbs paralysed by poliomyelitis, complete correction of knee flexion contracture is essential for maximal improvement in the ability to walk. A contracture of 15 to 50 degrees can be adequately corrected by a one-stage supracondylar femoral osteotomy, which is a simple procedure with a low morbidity. For more severe contractures, soft tissue release should be done first, but posterior capsulotomy should be avoided as far as possible to prevent posterior tibial subluxation.

\section{REFERENCES}

Abraham E, Verinder DGR, Sharrard WJW. The treatment of flexion contracture of the knee in myelomeningocele. J Bone Joint Surg [Br] 1977:59-B:433-8.

Bradford EH, Lovett RW. Treatise on orthopedic surgery. 2nd ed. London: Bailliere, Tindall and Cox, 1900:486.

Clark JMP. Muscle and tendon transposition in poliomyelitis In: Platt Sir H, ed. Modern trends in orthopaedics (2nd series). London: Butterworth, 1956:116-43.

Conner AN. The treatment of flexion contractures of the knee in poliomyelitis. J Bone Joint Surg [Br] 1970:52-B:138-44.

Keats S, Kambin P. An evaluation of surgery for the correction of knee-flexion contracture in children with cerebral spastic paralysis. $J$ Bone Joint Surg $[A m]$ 1962;44-A:1146-54.

Wilson PD. Posterior capsuloplasty in certain flexion contractures of the knee. J Bone Joint Surg 1929:11:40-58.

Yount CC. The rôle of the tensor fasciae femoris in certain deformities of the lower extremities. J Bone Joint Surg 1926;8:171-93. 